det, dass Frauen auf keinen Fall in die Ecke der so genannten „Quotenfrau“ gestellt, sondern einzig aufgrund ihrer Kompetenz und beruflichen Erfolge beurteilt werden wollen. Und in der Tat ist dies - wie schon ausgeführt - Grund und Ursache für die jüngsten Berufungen. Wenn aber gerade diese Frauen nicht aus der Selbstsicherheit des eigenen Erfolgs heraus sichtbar Engagement für Frauen in ihren jeweiligen Unternehmen zeigen, von wem wollen wir es dann erwarten? Wir können uns nicht einerseits über eine noch immer nicht ausreichende Zugkraft der Frauenförderung in Unternehmen beklagen, uns selbst aber mit dem Thema - in welcher Form auch immer nicht identifizieren wollen.

These 9: Ein nahezu ungelöstes Thema in Unternehmen scheint mir darüber hinaus die Frage zu sein, was Unternehmen mit den vielen bunten Köpfen, die nunmehr vermehrt gewonnen werden, denn nun anfangen sollen. Wissenschaftlich ist längst belegt, dass die Mehrung „bunter“ Köpfe im Unternehmen allein noch keinen Mehrwert darstellt. Vielmehr gilt es, sie so einzubinden und in ihrer Vielfalt zielgerichtet zu nutzen, dass ein Mehrwert für das Unternehmen und die jeweiligen Teams geschaffen wird. Hier können wir von unseren europäischen Nachbarn Vieles lernen, wie dies gelingen kann: Der Begriff Diversity-Management gilt in vielen unserer Nachbarländer als gescheitertes Konzept, stattdessen wird inzwischen über Inklusions- und über so genannte „Unsconscious Bias“-Projekte gesprochen, die stärker auf eben diese zielgerichtete Einbindung abstellen. Das wiederum setzt im Ergebnis einen Kulturwandel in Unternehmen voraus, der letztendlich nur dann erfolgreich sein kann, wenn er vom CEO getrieben breit durch das gesamte Unternehmen getragen wird. Wenn ein solch breit angelegter Kulturwandel in Unternehmen jedoch nicht gelingt, werden viele auf TopEbene rekrutierte Frauen sich in häufig noch sehr traditionell deutsch geprägten Unternehmen wiederfinden, in denen die
Verankerung und Nutzung von Andersartigkeit und Vielfalt nicht als Chance, sondern eher als Irritation wahrgenommen wird. In einem solchen Umfeld als Managerin zu reüssieren, kann dann bestenfalls mit großer Kraftanstrengung und enormem Beharrungsvermögen gelingen.

These 10: Last but not least gehört die aktive Auseinandersetzung mit Vorprägungen und Vorurteilen, denen sich Frauen vielfältig in Unternehmen ausgesetzt sehen, zu einem wesentlichen Element des beschriebenen Kulturwandels. Was in unseren Nachbarländern in vielfältigen „Unsconscious Bias“- Projekten verfolgt wird, ist die Schaffung von Transparenz hinsichtlich dieser Vorurteile und Vorprägungen auf allen Ebenen des Unternehmens. Damit soll Mitarbeitern, insbesondere aber auch den männlich dominierten Leitungsebenen eines Unternehmens geholfen werden, sich solcher Vorurteile, die wir natürlich alle besitzen, bewusst zu machen und aktiv mit ihnen umzugehen. Berichten insbesondere britischer Unternehmen folgend sind die Erfolge, die bei Anwendung solcher Trainings erzielt werden können, sehr erfolgversprechend und geeignet, zu weitreichenden Wandlungsprozessen in Unternehmen sehr positiv beizutragen. Es ist erfolgskritisch, die dargelegten Themen parallel anzugehen, um jenseits des „Köpfezählens“ weibliche Managerinnen erfolgreich in einer neuen und offeneren Unternehmenskultur zu verankern, die es nach meiner Überzeugung noch nicht häufig genug gibt. Dennoch - wenn Sie mir gestatten zu resümieren - ist das Glas aus meiner Sicht mehr als „halb voll“, es ist längst nicht mehr nur „halb leer“. Ich bin davon überzeugt, dass wir jenseits einer weiteren Verbesserung der Präsenz von Frauen auf allen Ebenen auf eine positive Öffnung und einen entsprechenden Kulturwandel in Unternehmen hinarbeiten sollten. Nur wenn beides gleichermaßen gelingt, werden wir uns alle hoffentlich recht bald in einer neuen Unternehmensrealität wiederfinden.

\title{
Frauenquote? Selbstverständlich
}

\section{Stephanie Bschorr}

Präsidentin Verband deutscher Unternehmerinnen (VdU) e.V., Berlin

Sehr geehrte Frau Präsidentin Pisal, sehr geehrter Herr Botschafter, sehr geehrter Herr Sattelberger, sehr geehrte Damen und Herren,

Sie alle werden es wissen: Die Gleichberechtigung von Mann und Frau ist schon in Artikel drei unseres Grundgesetzes und damit seit mehr als 60 Jahren festgeschrieben. Dieses Grundrecht betrachten wir heute als Selbstverständlichkeit. Damals war es überaus umstritten. So bedurfte es allein mehrerer Jahrzehnte und zahlreicher Gesetzesänderungen, bis auch nur das in der Bundes- republik Deutschland geltende Recht diesem Grundrecht entsprach.

Darüber, wie lange es gedauert hat oder vielleicht sogar noch dauert, dieses Grundrecht in den Köpfen zu verankern, lässt sich nur spekulieren. Seitdem es das Grundrecht der Gleichberechtigung von Mann und Frau gibt, gibt es aber auch die Erwartung der Chancengleichheit von Frauen und Männern - und zwar in allen Lebensbereichen. Und, keine Frage, es ist viel erreicht. Aber ein Feld liegt noch, wenn ich das so sagen darf, nahezu unbestellt vor uns: die Chancengleichheit von Frauen und Männern in den Führungsebenen und Aufsichtsgremien der deutschen Unternehmen.

Seit elf Jahren bereits blicken wir auf die Vereinbarung zwischen der Bundesregierung und den Spitzenverbänden der deutschen Wirtschaft zur Förderung der Chancengleich- 
heit von Frauen und Männern in der Privatwirtschaft. Seit elf Jahren sind die erzielten Fortschritte im Bereich der Frauen in Führungspositionen und Aufsichtsgremien sehr überschaubar. Von dem erhofften, ja erwarteten Kulturwechsel in den Vorständen und Aufsichtsräten der deutschen Unternehmen kann keine Rede sein. Vielmehr befinden sich viele von uns immer noch im Stadium des Austauschs von Argumenten. Da gibt es die Gruppe derer, die durch gesetzliche Regelungen ihre unternehmerische Entscheidungsfreiheit gefährdet sieht, die Gruppe derer, die sich nur sehr zögerlich einer modernen Unternehmens- und Führungskultur öffnet, die Gruppe derer, die alte Führungsstile verteidigt, und das mit eher fragwürdigen Argumenten. Wie oft ist mir insbesondere in den letzten Wochen vor dem Hintergrund der Berliner Erklärung und des SPD-Gesetzentwurfs zur Quote erklärt worden, dass eine Frauenquote zu einem Absinken des Qualifikationsniveaus in den Gremien führte. Wie oft ist mir erklärt worden, dass eine Quote die unternehmerische Freiheit bei der Besetzung von Positionen abschaffe. Wie oft ist mir erklärt worden, dass eine Quote die hervorragend qualifizierten Frauen diskreditierte.

Dem gegenüber stehen zahlreiche Untersuchen, die belegen, dass gemischte Teams deutlich effizienter sind. Dem gegenüber stehen die positiven Erfahrungen, die in anderen europäischen Ländern mit Frauenquoten gemacht wurden. Und dem gegenüber steht auch die jüngste freiwillige Initiative der DAX-Konzerne aus dem Frühjahr 2011, im Rahmen derer angekündigt wurde, bis 2020 den Anteil von Frauen in den Führungsgremien auf bis zu 35 Prozent steigern zu wollen. Eine Ankündigung, die von der Bundeskanzlerin damals mit "Seien Sie kreativ oder wir werden kreativ sein“ kommentiert wurde.

Demnach sind sich sowohl die führenden Wirtschaftsvertreter wie auch die Politik einig, dass der Anteil von Frauen in Führungspositionen und Aufsichtsräten erhöht werden muss. Umso mehr verwundert es, dass über den Weg dahin so erbittert gestritten wird. Das hat aus meiner Sicht folgenden Grund: Die Frauenquote wird in Deutschland hoch emotional diskutiert. Es geht schon längst nicht mehr um Sachargumente. Es geht um das Rollenverständnis und die Weltbilder mancher Wirtschaftsführer, die durch eine Quotenregelung ins Wanken geraten. Und es geht um die Verabschiedung von Strukturen: von Strukturen, die erprobt und bewährt sind, von Strukturen, die Sicherheit gegeben haben und immer noch geben, von Strukturen, die - wie ich letzte Woche las - als verantwortlich gelten für den einzigartigen Erfolg der deutschen Wirtschaft. Aber: Die Zeiten ändern sich und die Unternehmen in Deutschland müssen neue Wege gehen - gerade auch, um wirtschaftlich erfolgreich zu bleiben.

Einen Weg, um den Anteil von Frauen in Führungspositionen zu steigern, und zwar den Weg, den der VdU beschritten hat, möchte ich Ihnen skizzieren: Als der VdU vor mehr als drei Jahren als einziger Wirtschaftsverband beschlossen hatte, eine Quote von 40 Prozent für die Aufsichtsgremien der Unternehmen zu fordern, war es ihm ein wichtiges Anliegen, diese für Unternehmerinnen nicht selbstverständliche politische Forderung auch konstruktiv zu begleiten. Aus diesem Grund hat der VdU mit Unterstützung der Bundesinitiative "Gleichstellung von Frauen in der Wirtschaft“ das Projekt „Stärkere Präsenz von Frauen in Aufsichtsgremien“ initiiert.

Dieses Projekt hat zwei Elemente: Zum einen bereiten wir bis 2013150 weibliche TOP-Führungskräfte durch entsprechende Seminare auf die Übernahme eines Mandats in einem Aufsichtsgremium vor. Inzwischen haben nahezu 90 Frauen an unseren Seminaren teilgenommen. Eine von ihnen ist mittlerweile Aufsichtsratsmitglied eines DAX-Unternehmens. Wir sind optimistisch, dass in nächster Zeit weitere Frauen folgen werden.

Die Kandidatinnen für die Seminare, die von PriceWaterhouseCoopers und einer erfahrenen Journalistin und Medientrainerin vom NDR durchgeführt werden, werden von einer hochrangigen Jury ausgesucht. Dieser Jury gehören die ehemalige Bundesjustizministerin, Frau Brigitte Zypries, Herr Prof. Burkhard Schwenker von Roland Berger Strategy Consultants, Herr Dr. Axel Smend von der Deutschen Agentur für Aufsichtsräte und die jeweils amtierende VdUPräsidentin an.

Zum anderen unterstützen wir mit Hilfe einer Datenbank Unternehmen bei der Suche nach geeigneten Kandidatinnen für ihr Aufsichtsgremium. Gerade vor dem Hintergrund der immer wiederkehrenden Behauptung, dass vakante Positionen nicht mit Frauen besetzt werden könnten, da es keine geeigneten Kandidatinnen gäbe, war es dem VdU ein wichtiges Anliegen, geeignete Kandidatinnen sichtbar zu machen. Mittlerweile haben wir in unserer Datenbank fast 500 Frauen erfasst. Sie alle haben beeindruckende Lebensläufe. Sie alle können jederzeit ein Mandat übernehmen.

Unsere Erfahrungen der letzten zwei Jahre zeigen aber auch, dass es eine Zeit braucht, bis sich bei der Auswahl von Kandidatinnen und Kandidaten für Aufsichtsgremien neue Wege etablieren. Der bisher übliche Weg, nämlich Personen als Kandidaten zu nominieren, die persönlich bekannt sind, wird sich zunehmend aufweichen. Angesichts der geforderten fundierten fachlichen Erfahrungen, der Fähigkeiten und Branchenkenntnisse werden zunehmend Beratungsgesellschaften und auch der VdU angefragt werden, wenn es gilt ein Portfolio an hoch qualifizierten Kandidatinnen zu präsentieren.

Trotz aller Einigkeit von Politik und Wirtschaft darüber, dass ein höherer Anteil von Frauen in Führungspositionen erforderlich ist, trotz all der entsprechenden Willensbekundungen aus der Wirtschaft, trotz all der Bemühungen, dieses Ziel durch Projekte, wie das VdU-Projekt „Stärkere Präsenz von Frauen in Aufsichtsgremien“ zu unterstützen, muss ich leider sagen, die vergangenen elf Jahre haben gezeigt: Der freiwillige Weg ist gescheitert. Ich bedauere das sehr. Gerade mir als Unternehmerin liegt der Ruf nach gesetzlichen Regelungen grundsätzlich eher fern. In diesem Fall aber plädiere 
ich für eine gesetzliche Regelung zur Steigerung des Anteils der Frauen in Führungspositionen, plädiere ich für eine gesetzlich geregelte Frauenquote - zumindest vorübergehend.

Ich bin fest davon überzeugt, dass wir spätestens in der nächsten Legislaturperiode und mit Unterstützung aus der EU den Weg der Freiwilligkeit und damit auch des Schneckentempos bei der Realisierung der Frauenquote verlassen werden. Und ich bin davon überzeugt, dass wir dann in
Deutschland endlich realisieren werden, dass eine gesetzliche Frauenquote nicht nur verfassungsrechtlich möglich, sondern auch wirtschaftlich sinnvoll ist. Vielleicht ergeht es der Frauenquote in Zukunft dann auch einmal so wie der Gleichberechtigung von Mann und Frau, nämlich, dass wir sie als Selbstverständlichkeit empfinden. Ich jedenfalls, würde es ihr, unserer Gesellschaft und auch unseren Wirtschaftunternehmen wünschen.

\section{Zähne für den Tiger!}

\section{Sigrun Mast, Maître en Droit}

Rechtsanwältin, Hamburg

Auch ich begrüße Sie herzlich zu „Wie soll der Wandel gelingen “. Ich heiße Sigrun Mast, bin Mitglied im Deutschen Juristinnenbund und als Rechtsanwältin in der Wirtschaftsprüfungsgesellschaft RöverBrönnerSusat tätig.

Birgit Kersten, die maßgeblich beim Deutschen Juristinnenbund das Projekt „Aktionärinnen fordern ein “ begleitet, hat mich im Anschluss an einen internen Workshop gefragt, ob ich im Rahmen einer Veranstaltung ein kurzes Statement abgeben könne. Anlass war insbesondere mein Besuch des Euroforum-Seminars „Der zertifizierte Aufsichtsrat“ in 2011.

Das Seminar „Der zertifizierte Aufsichtsrat“ ist ein sechstägiges Seminar. Es gibt einige Übereinstimmungen mit dem Projekt des Verbands deutscher Unternehmerinnen „Wahrnehmung von Mandaten in Aufsichtsgremien“. Beim Euroforum gibt es aufgrund der anderen Zielgruppe kein Kommunikations- und Medientraining. Vielmehr ist der rechtliche Schwerpunkt verstärkt. Zusätzlich existieren ein ausführliches bilanzielles Modul und ein Abschlusstest. Nun, in allen wesentlichen Punkten stimmen beide Seminare überein. Allerdings hat sich Birgit Kersten in einer weiteren Mail gewünscht, dass ich die Frage „Wie soll der Wandel gelingen?“ aus meinem Arbeitsbereich und meiner persönlichen Sicht beantworte. Ok. Zunächst: Wo stehen wir?

Norwegen hat bereits eine Quote von 40 Prozent für Frauen in Aufsichtsräten eingeführt - norwegischen Aufsichtsräten. Allerdings kontrollieren norwegische Aufsichtsräte die Geschäftsführung nicht nur, sondern nehmen Geschäftsführungsaufgaben wahr. Deutsche Aufsichtsräte sind Überwachungsorgane. Wenn wir über einen Wandel reden, müssen wir uns fragen, ob wir einen norwegischen Wandel wollen; dann heißt es: Frauen in Führungspositionen. In Norwegen ist dieser rechtlichen Umsetzung eine hitzige gesellschaftliche Diskussion vorangegangen. Wir stecken mitten in dieser gesellschaftlichen Diskussion. Die gesellschaftliche Forderung ist die nach einer gesetzlichen Quote für Frauen in Aufsichtsräten.

Doch wie hängen Gesellschaft und Recht zusammen? Teils prägt die Gesellschaft das Recht, teils prägt das Recht die Gesellschaft. Für eine umfassende gesetzliche Änderung bedarf es einer weitgehenden gesellschaftlichen Akzeptanz. Diese fußt auf einer Notwendigkeit des Wandels - dem Sollen. Eine Mehrheit muss die Konsequenzen, die hieraus erwachsen, auch wollen. Die demografische Entwicklung und der daraus resultierende Fachkräftemangel führen zu einer solchen Notwendigkeit des Wandels. Glauben wir aktuellen Umfragen bejahen immer mehr Deutsche eine Frauenquote für Führungspositionen. Diese stellt einen Teil des Wandels dar. Meine weiteren Ausführungen beziehen daher sowohl das aktuelle Recht als auch das gesellschaftliche Sein, Wollen und Sollen mit ein.

Nehmen wir zunächst das praktische Beispiel des Seminars „Der zertifizierte Aufsichtsrat“. Wer besucht das „neue“ Seminar „Der zertifizierte Aufsichtsrat“ und warum? Das Seminar wird von Aufsichtsräten und Beiräten, von Geschäftsführern, Vorständen und internen und externen Beratern besucht. Es war ein kleiner Kreis von knapp über zehn Teilnehmern, davon drei Frauen. Alle interessierte die aktuelle Rechtslage. Einige hatten praktische Fragen aus dem Alltag. Aufsichtsräte und deren Berater sind seit der Wirtschaftskrise an diesen Seminaren interessiert. Die Frage der Aufsichtsratshaftung spielt eine immer stärkere Rolle. Spätestens seit dem Urteil im Fall ARAG-Garmenbeck. Danach muss der Aufsichtsrat Schadensersatzansprüche gegen den Vorstand verfolgen, wenn er nach eigener Prüfung von einer Schadenersatzpflicht des Vorstands ausgeht. Verstärkt fragten die Teilnehmer auch nach den wichtigsten Konditionen für eine DirectorsAndOfficers-Versicherung. Ein Vorstandsmitglied gab zu, dass seine Aufsichtsräte verstärkt Fragen stellen, bei denen er sich fragt, ob sie diese Fragen stellen müssen und dürfen.

Inwieweit ist der "Wandel“ bei diesem Seminar Thema? Rechtlich wird der Wandel durch den Deutschen Corporate Governance Kodex auf Ebene der Besetzung von Führungspositionen und des Aufsichtsrates thematisiert. Bei börsennotierten Gesellschaften erklären Vorstand und Aufsichtsrat jährlich, ob den Empfehlungen des Deutschen Corporate Governance Kodexes entsprochen wurde oder welche Empfehlungen nicht angewendet wurden oder werden und warum nicht. Der Deutsche Corporate Governance Kodex 